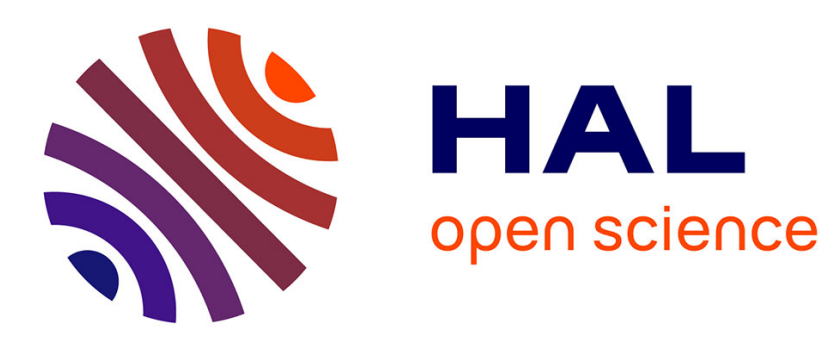

\title{
Assessing impact force localization by using Particle Swarm Optimization algorithm
}

Abdelali El-Bakari, Abdellatif Khamlichi, Eric Jacquelin, Rachid Dkiouak

\section{To cite this version:}

Abdelali El-Bakari, Abdellatif Khamlichi, Eric Jacquelin, Rachid Dkiouak. Assessing impact force localization by using Particle Swarm Optimization algorithm. Journal of Sound and Vibration, 2014, 333 (6), pp.1554-1561. 10.1016/j.jsv.2013.11.032 . hal-01417382

\section{HAL Id: hal-01417382 \\ https://hal.science/hal-01417382}

Submitted on 15 Dec 2016

HAL is a multi-disciplinary open access archive for the deposit and dissemination of scientific research documents, whether they are published or not. The documents may come from teaching and research institutions in France or abroad, or from public or private research centers.
L'archive ouverte pluridisciplinaire HAL, est destinée au dépôt et à la diffusion de documents scientifiques de niveau recherche, publiés ou non, émanant des établissements d'enseignement et de recherche français ou étrangers, des laboratoires publics ou privés. 


\title{
Assessing impact force localization by using Particle Swarm Optimization algorithm
}

\author{
Abdelali El-Bakari, \\ Mechanics and Civil Engineering Laboratory, FST, Tangier 91001, Morocco \\ Email: ab_elbakari@yahoo.fr
}

*Abdellatif Khamlichi,

Telecommunications Systems Laboratory, FS, M'hannech II, Tetouan 93002, Morocco

Phone: +212679148263

Email: khamlichi7@yahoo.es

\section{Eric Jacquelin,}

Université de Lyon, F-69622, Lyon ; IFSTTAR, LBMC, UMR_T9406, Bron ; Université Lyon 1, Villeurbanne, France.

Email: eric.jacquelin@univ-lyon1.fr

\section{Rachid Dkiouak,}

Mechanics and Civil Engineering Laboratory, FST, Tangier 91001, Morocco

Email: rdkiouak@hotmail.com

\begin{abstract}
This paper focuses on the inverse problem regarding force localization in the case of non punctual impacts occurring on elastic beams. Following the identification approach proposed to solve this problem and which is based on the reciprocity theorem, the impact location characteristics were determined by using Particle Swarm Optimization algorithm. To eliminate numerical trouble due to the trivial solutions appearing in this formulation, the fitness function was customized by introducing a set of weighting coefficients. Four different formulations of the fitness function were considered and their performances with regards to the number of sensors used and their positions were analyzed. This enabled to select the best combination of weighting coefficients to be used in the context of an impact force localization process based on Particle Swarm Optimization technique. Three sensors were found to be required and comparison with Genetic Algorithm has revealed effectiveness of the proposed method in terms of accuracy and computational time.
\end{abstract}

Key words: Inverse problem, force localization, reciprocity theorem, fitness function, Particle Swarm Optimization

* Corresponding author 


\section{Introduction}

Structures endure the risk of various impact events. Severe degradation of structural strength or stiffness might result from impact-induced damages. In order to keep up the integrity and operation safety of vital structures, detecting internal damages after occurrence of an impact event is of great importance. Classic non-destructive inspection techniques such as ultrasound or X-ray based methods have been widely used. However, these conventional techniques do not permit on-line inspection which is quite essential in some applications. As a novel inspection approach, structural health monitoring has the capacity of dealing with such concerns. In this methodology sensors and damage check-up algorithms can be implemented on the target structures, enabling for structural integrity to be automatically supervised by using real-time information.

Under impact circumstances, information about the impact force magnitude can enable assessing the damage pattern and extent affecting structures. To perform such advanced damage quantifying assignment, the external impact force should be first accurately identified. In many practical situations, direct measurement of impact forces is not easy to carry out. However, indirect methods that are based on the measured dynamic response at discrete points by some judiciously implemented sensors can provide complete information regarding the position and magnitude of these sources. This needs nevertheless performing solution of an inverse problem [1].

In case of linear elastic structures such as beams or plates, identification of impact characteristics can be implemented through using a structural model [2,3]. This can be constructed analytically, by means of the finite element method or by experimental identification procedures. When the impact can be assumed to be punctual and the impact location is known such as in contact problems, the response functions between the impact point and the sensors placed at known positions allow, by regularized deconvolution, to reconstruct the force signal. When the point of impact is unknown, the force location can be found through an inverse formulation. This can be achieved through a minimization technique between the measured and calculated responses in order to reconstruct the impact characteristics: force point location and force temporal signal [4].

Force location is extremely difficult to tackle by using the inverse methods. One cannot just consider adding the force point coordinates as extra unknown parameters to the problem, because these positions intervene as implicit parameters, in contrast with the force history values which are explicit. Iterations are required then for solution of positions unknowns 
which give rise to a numerical conflict situation. Treating all the force history values to be implicit parameters is not a good option as it would increase dramatically the number of unknowns and increase the computational cost. Therefore, a hybrid scheme decoupling the problem in two parts was introduced [5]. This scheme involves on one hand force reconstruction by regularized deconvolution and on the other hand implicit parameter identification through solution of a non linear mathematical program which provides force location.

The problem is more complex when the impact force is distributed over an area of the structure surface that cannot be assumed to be punctual, because it involves identifying multiple forces and not only a single concentrated force. Even when these forces can be supposed to be uniform, unknown parameters that represent the extent of the impacted area come out as additional implicit unknowns.

To perform exploration of impact force location, various effective methods have been proposed in the literature. For one-dimensional beam-like structures force localization has been investigated in [6]. Martin and Doyle [7] have described how to find the location of an impact force using dynamic response measurements. They proposed a solution procedure using the spectral element method with a stochastic iterative search. Experimentally measured acceleration responses from two frame structures were used to achieve force localization by minimizing a fitness function. A Genetic Algorithm (GA) was used to guess iteratively the minimum through monitoring the actual error associated to a given sampling generation. The process enabled to discriminate between good and bad guesses and gave at convergence the correct impact location. An alternative technique which employs the arrival time of each frequency component of a pulse was proposed by Tribikram et al. [8] and Liang et al. [9]. The main problem with this technique is that anisotropy of the structure should be taken into account and measurement of small arrival times could suffer uncertainties.

In this work, we propose a technique based on separation between the localization and reconstruction problems in order to determine the impact characteristics in two steps. Elastic structures subjected to non punctual impacts for which the force can be assumed to be uniformally distributed over the impact zone are considered. The localization problem is solved as a minimization problem in the form of a non linear mathematical program providing the impact forces positions. Since these positions are implicit unknowns, the best suited minimization procedures are those of evolutionary type. Investigations clearly showed that the Particle Swarm Optimization (PSO) approach always provided a better solution than the GA based approaches [10]. Due to its high performance in terms of accuracy and robustness, PSO 
algorithm will be considered in the following. Focus will be done on the particular role of the fitness function. This last will be derived from the reciprocity Maxwell-Betti theorem which is valid for any elastic structure. Since the direct equation obtained by this theorem has trivial solutions that impede the optimization process to converge to the practical solution, the fitness function has to be customized [4]. Three novel formulations of the modified fitness function that enable removing the trivial solutions are introduced and investigated. The aim is to determine the best choice with regard to PSO convergence requirement, as well as the number of sensors used, positions of these sensors and computational cost.

\section{Materials and methods}

\subsection{Formulation of the direct problem}

Although the problem can be stated for any elastic structural system, to fix ideas, we consider only a simplified model in the form of a beam having a rectangular section as shown in figure 1. The beam is assumed to be simply supported on both ends and having length $\mathrm{L}$, width $\mathrm{b}$ and height $\mathrm{e}$. It is assumed to be made from a homogeneous and isotropic elastic material with Young's modulus E and density $\rho$. The applied force modelling impact is assumed to result from a uniformly distributed pressure, $\mathrm{p}$, which is applied on a rectangular patch $\left[\mathrm{s}_{0}-\mathrm{u}_{0}, \mathrm{~s}_{0}+\mathrm{u}_{0}\right]$ as shown in figure 1 . The pressure rectangle is assumed to be centered on $\mathrm{s}_{0}$ and having the length $2 \mathrm{u}_{0}$.

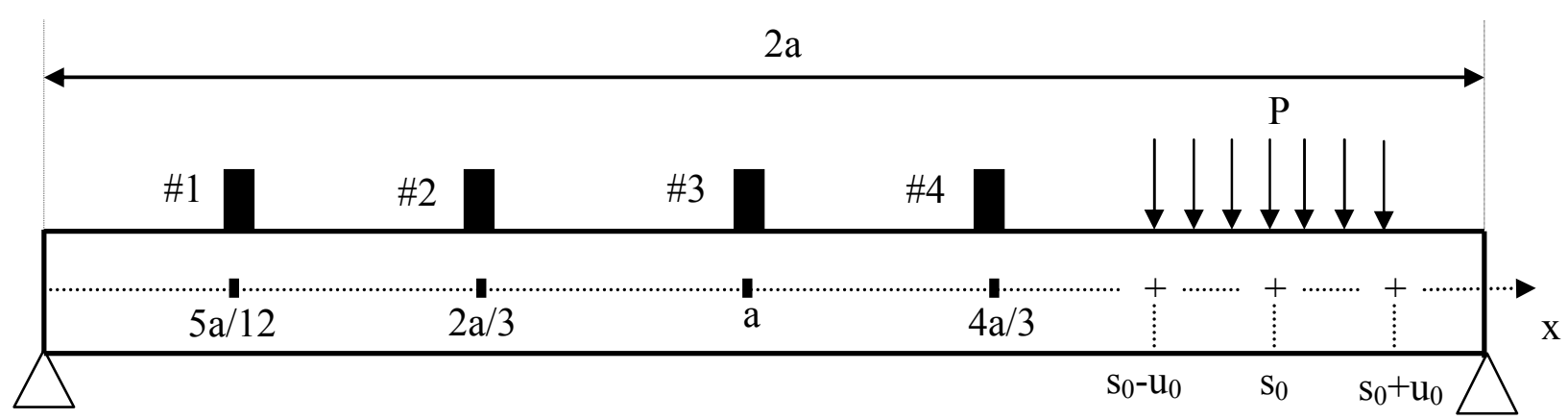

Fig. 1. An elastic beam having a uniform rectangular cross section and loaded with a distributed uniform pressure; positions of four sensors are indicated

The dynamic response is considered for a point having a given abscissa referenced with respect to the origin chosen to be the left extremity of the beam while the axis is oriented 
towards the right. Four strain gauge sensors will be investigated, the sensors positions are shown on figure 1 with their labels and relative abscissas.

Under the action of an impulse pressure, the transient dynamic response $\mathrm{y}$ in terms of displacement, acceleration or strain can be measured by a proper sensor located at a given abscissa. Considering the time interval to be of length $\mathrm{N}$, after operating sampling, the discrete representation of the linear system with multiple degrees of freedom that models the beam dynamic behavior writes as follows [4]

$$
\mathrm{Y}=\mathrm{HP}
$$

with

$$
\begin{aligned}
Y & =\left[\begin{array}{lll}
y(1) & y(2) & y(N)
\end{array}\right]^{t} \\
P & =\left[\begin{array}{llll}
p(1) & p(2) & p(N)
\end{array}\right]^{t} \\
H & =\left[\begin{array}{cccc}
h(1) & 0 & \cdots & 0 \\
h(2) & h(1) & \ddots & \vdots \\
\vdots & \vdots & \ddots & 0 \\
h(N) & h(N-1) & \cdots & h(1)
\end{array}\right]
\end{aligned}
$$

where $\mathrm{y}(\mathrm{k})$ is the discrete output as observed by sensors and $\mathrm{p}(\mathrm{k})$ the discrete pressure input, $\mathrm{h}$ is a discrete response function approximating the considered linear system and $\mathrm{H}$ is the Toeplitz like transfer matrix connecting the pressure $p$ to the measured signal $y$.

The matrix $\mathrm{H}$ can be constructed analytically, but can also be obtained by means of the finite element method or by using experimental identification procedures. So, the model defined by equation (1) is more general and should not be thought of to be restricted to the simplified case considered here for the purpose to abridge presentation. It is assumed on the other hand that the notation $\mathrm{H}$ is reserved to the response in terms of displacement.

The dynamic Euler Bernoulli beam equation under simple bending can be solved by means of modal superposition method and Duhamel's integral [11]. Considering the case of a pinnedpinned beam, the mode number $\mathrm{m}\left(\mathrm{m} \in \square^{*}\right)$ is given by $\left\{\omega_{\mathrm{m}}, \varphi_{\mathrm{m}}(\mathrm{x})\right\}$ with $\omega_{\mathrm{m}}=\frac{\mathrm{m}^{2} \pi^{2}}{\mathrm{~L}^{2}} \sqrt{\frac{\mathrm{EI}}{\rho \mathrm{S}}}$ the circular eigenfrequency and $\varphi_{\mathrm{m}}(\mathrm{x})=\sin \left(\frac{\mathrm{m} \pi \mathrm{x}}{\mathrm{L}}\right)$ the associated mode shape normalized according to $\max _{\mathrm{x}}\left\{\varphi_{\mathrm{m}}(\mathrm{x})\right\}=1$. Denoting $\Delta \mathrm{t}$ the time step used in time discretisation, $\mathrm{M}$ the 
number of truncated modes retained to synthesize the beam dynamical response from modal expansion, the impulse response function giving the transverse displacement of the beam section having abscissa $a_{i}$ is shown to be given as

$$
\mathrm{H}_{\mathrm{kj}}\left(\mathrm{s}_{0}, \mathrm{u}_{0}, \mathrm{a}_{\mathrm{i}}\right)=-\frac{4 \pi}{\rho \mathrm{SL}^{2}} \sum_{\mathrm{m}=1}^{\mathrm{M}} \sin \left(\frac{\mathrm{m} \pi \mathrm{s}_{0}}{\mathrm{~L}}\right) \sin \left(\frac{\mathrm{m} \pi \mathrm{u}_{0}}{\mathrm{~L}}\right) \sin \left(\frac{\mathrm{m} \pi \mathrm{a}_{\mathrm{i}}}{\mathrm{L}}\right) \mathrm{g}\left(\omega_{\mathrm{m}}, \xi_{\mathrm{m}},(\mathrm{j}-\mathrm{k}) \Delta \mathrm{T}\right)
$$

with

$$
g\left(\omega_{\mathrm{m}}, \xi_{\mathrm{m}},(\mathrm{j}-\mathrm{k}) \Delta \mathrm{T}\right)=\frac{\mathrm{mexp}\left(-\xi_{\mathrm{m}} \omega_{\mathrm{m}}(\mathrm{j}-\mathrm{k}) \Delta \mathrm{T}\right)}{\omega_{\mathrm{m}} \sqrt{1-\xi_{\mathrm{m}}^{2}}} \sin \left(\omega_{\mathrm{m}} \sqrt{1-\xi_{\mathrm{m}}^{2}}(\mathrm{j}-\mathrm{k}) \Delta \mathrm{T}\right)
$$

where $\xi_{\mathrm{m}}$ is the damping ratio associated to mode number $\mathrm{m}$.

\subsection{Fitness function associated to the localization problem}

Direct inverse methods for solving the localization problem of impact are known to be computationally very intensive because an iterative approach is usually required [9]. In the following the Maxwell-Betti theorem based method is used [4]. Locating the impact zone for the beam problem considered in this study consists in finding the impact patch center position $\mathrm{s}_{0}$ and parameter $\mathrm{u}_{0}$ which defines the extent of the impacted zone. The responses measured by strain sensors placed at points having respectively the abscissa $a_{i}$ and $a_{j}$ can be expressed under the following form

$$
\mathrm{Y}_{\mathrm{i}}=\mathrm{G}_{\mathrm{i}}\left(\mathrm{s}_{0}, \mathrm{u}_{0}\right) \mathrm{P} \quad \mathrm{Y}_{\mathrm{j}}=\mathrm{G}_{\mathrm{j}}\left(\mathrm{s}_{0}, \mathrm{u}_{0}\right) \mathrm{P}
$$

where $\mathrm{G}_{\mathrm{i}}\left(\mathrm{s}_{0}, \mathrm{u}_{0}\right)=\mathrm{G}\left(\mathrm{s}_{0}, \mathrm{u}_{0}, \mathrm{a}_{\mathrm{i}}\right)$ and $\mathrm{G}_{\mathrm{j}}\left(\mathrm{s}_{0}, \mathrm{u}_{0}\right)=\mathrm{G}\left(\mathrm{s}_{0}, \mathrm{u}_{0}, \mathrm{a}_{\mathrm{j}}\right)$ are respectively the transfer functions between the input pressure and the measured axial deformation at the upper fiber of the beam by the sensors placed at $a_{i}$ and $a_{j}$. These sensors can be any given pair selected from the set $\{\# 1, \# 2, \# 3, \# 4\}$ as shown in figure 1 .

In the case of a beam having a uniform rectangular section of height e, the transfer function which is related to the normal longitudinal strain of the upper beam fiber and denoted $G$ is obtained from the displacement transfer function $\mathrm{H}$, given by equation (4), as $\mathrm{G}=-(\mathrm{e} / 2) \mathrm{H}$. 
The commutative property $\mathrm{G}_{\mathrm{i}}\left(\mathrm{s}_{0}, \mathrm{u}_{0}\right) \mathrm{G}_{\mathrm{j}}\left(\mathrm{s}_{0}, \mathrm{u}_{0}\right)=\mathrm{G}_{\mathrm{j}}\left(\mathrm{s}_{0}, \mathrm{u}_{0}\right) \mathrm{G}_{\mathrm{i}}\left(\mathrm{s}_{0}, \mathrm{u}_{0}\right)$ resulting from MaxwellBetti theorem which apply for any elastic system yields as shown in [4] to the following identity

$$
\mathrm{G}_{\mathrm{j}}\left(\mathrm{s}_{0}, \mathrm{u}_{0}\right) \mathrm{Y}_{\mathrm{i}}=\mathrm{G}_{\mathrm{i}}\left(\mathrm{s}_{0}, \mathrm{u}_{0}\right) \mathrm{Y}_{\mathrm{j}}
$$

The big merit of equation (7) is that it does not involve the force history vector $\mathrm{P}$, so knowledge of the force signal is not needed to find the impact zone characteristics $\left(\mathrm{s}_{0}, \mathrm{u}_{0}\right)$ as these are provided by solution of this implicit equation independently of vector $\mathrm{P}$. The process of finding $\left(\mathrm{s}_{0}, \mathrm{u}_{0}\right)$ works according to the statement that giving the recorded strain responses $\mathrm{Y}_{\mathrm{i}}, \mathrm{Y}_{\mathrm{j}}$ and the locations where these are sensed, denoted respectively by indices $\mathrm{i}$ and $\mathrm{j}$, equation (7) will be satisfied if the intervening transfer functions $\mathrm{G}_{\mathrm{i}}\left(\mathrm{s}_{0}, \mathrm{u}_{0}\right)$ and $\mathrm{G}_{\mathrm{j}}\left(\mathrm{s}_{0}, \mathrm{u}_{0}\right)$ are those which are associated to the actual impact position [4]. One should however notice from equation (4), that both members of equation (7) will vanish automatically for $\mathrm{s}=0$ or $\mathrm{s}=\mathrm{L}$ or $\mathrm{u}=0$, yielding thus an infinity of trivial solutions.

To avoid these parasitic solutions which impede obtaining the unique physical solution satisfying equation (7), some regularization of the problem is required. To perform this, one should remove the trivial solutions $\mathrm{s}=0$ or $\mathrm{s}=\mathrm{L}$ or $\mathrm{u}=0$ by transforming equation (7) into the following nonlinear mathematical program

$$
\left(s_{0}, u_{0}\right)=\underset{(s, u)}{\operatorname{Arg} \min }\left\{\phi(s, u)=\sum_{i=1}^{N_{s}} \sum_{j=1, j \neq i}^{N_{s}} \frac{1}{\alpha_{i j}}\left\|G_{j}(s, u) Y_{i}-G_{i}(s, u) Y_{j}\right\|^{2}\right\}
$$

where $\mathrm{N}_{\mathrm{s}} \geq 2$ denotes the number of sensors used and $\alpha_{\mathrm{ij}}$ are some weights.

For the material and geometric data given in the fourth section, Figure 2 gives the objective function which corresponds to $\alpha_{\mathrm{ij}}=1$, and in the case where the two sensors $\# 2$ and \#4 are used. This figure shows that the points satisfying: $\mathrm{s}=0$ or $\mathrm{s}=\mathrm{L}$ or $\mathrm{u}=0$, constitute clearly trivial solutions of the minimization problem. The exact solution which corresponds to the given data in the direct problem taken to be $\left(\mathrm{s}_{0}=0.417 \mathrm{~m}, \mathrm{u}_{0}=0.0417 \mathrm{~m}\right)$ is shown in figure (2b) as a star. This solution is out of reach directly by any minimization procedure of the fitness function $\phi$ for the case $\alpha_{i j}=1$. This states the necessity for this function to be transformed in order to remove all the trivial solutions. 


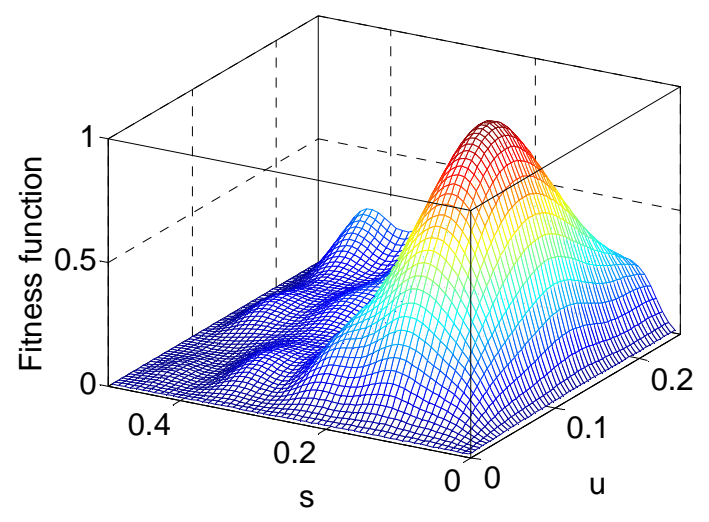

(a)

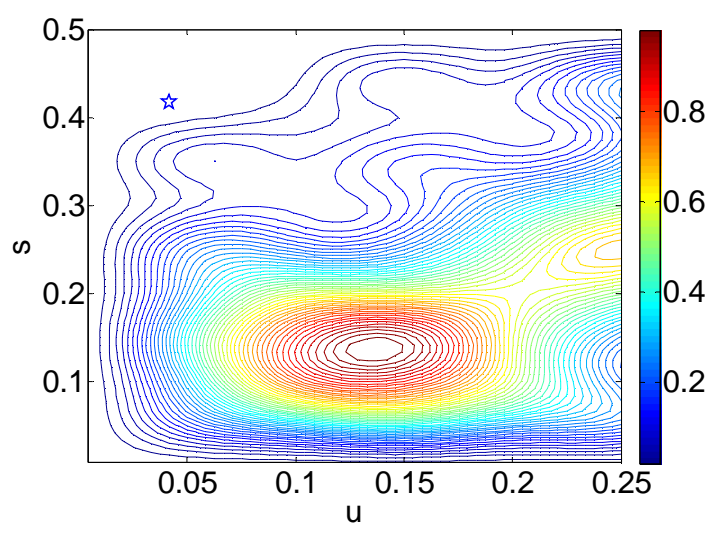

(b)

Fig. 2. The fitness function $\phi$ for $\alpha_{\mathrm{ij}}=1$; (a) the unmodified fitness function surface, (b) contours of the unmodified fitness function

Equation (8) will admit the physical solution $\left(\mathrm{s}_{0}, \mathrm{u}_{0}\right)$ as the unique solution if the introduced weights are able to eliminate the parasitic solutions. Their role consists in transforming the loss function $\sum_{i=1}^{N_{s}} \sum_{j=1, j \neq i}^{N_{s}}\left\|G_{j}(s, u) Y_{i}-G_{i}(s, u) Y_{j}\right\|^{2}$ which is strictly equivalent to equation (7), by enlarging its values for $(\mathrm{s}, \mathrm{u})$ that are close to the boundary set: $\mathrm{s}=0$ or $\mathrm{s}=\mathrm{L}$ or $\mathrm{u}=0$. The two parameters that define the impact location $\mathrm{s}_{0}$ and $\mathrm{u}_{0}$ can then be obtained as the unique minimum of the loss function $\phi$. Yen and $\mathrm{Wu}[4]$ have proposed the following expressions for the weights $\alpha_{i j}=\left\|G_{i}(s, u) Y_{j}\right\|^{2}$.

Many algorithms can be used to solve the mathematical problem as defined by equation (8). The most performant ones are however those derived form evolutionary algorithms, since they can deal more easily with situations where the fitness function is not explicit and containing implicit unknown parameters. In this paper the optimization procedure is based on the PSO algorithm [12], known to be quite efficient in these conditions.
Case 1
Case 2
Case 3
Case 4

$$
\left\|G_{i}(s, u) Y_{j}\right\|^{2} \quad\left\|G_{i}(s, u)\right\|^{2}+\left\|G_{j}(s, u)\right\|^{2} \quad\left\|G_{i}(s, u)\right\|+\left\|G_{j}(s, u)\right\| \quad\left\|G_{i}(s, u) Y_{j}\right\|^{2}+\left\|G_{j}(s, u) Y_{i}\right\|^{2}
$$

Table 1: Considered expressions for the weight coefficients $\alpha_{i j}$ 
To solve the localization problem and to assess the effect due to a particular choice of the weight coefficients $\alpha_{\mathrm{ij}}$ on convergence of the PSO algorithm, three novel expressions will be introduced for these. They are given by the last three columns of Table 1, while the first column of this Table recalls the Yen and $\mathrm{Wu}[4]$ weight coefficients.

Because of periodicity of equation (8) with regards to $s_{0}$ and $u_{0}$ which can be seen easily form equation (4), to stabilize the minimization procedure and obtain the practical solution, the unconstrained mathematical program defined by equation (8) is completed by adding the following geometric constraints

$$
\begin{aligned}
& 0 \leq \mathrm{u}_{0} \leq \mathrm{L} / 2 \\
& \mathrm{u}_{0} \leq \mathrm{s}_{0} \leq \mathrm{L}-\mathrm{u}_{0}
\end{aligned}
$$

According to equation (8), the cost function depends also on the number of sensors and their positions. The influence of the weights $\alpha_{i j}$ on the PSO algorithm performance will be analyzed then in conjunction with the sensor network configuration. The objective is to determine sensitivity of the procedure with respect to various sensors configurations. Convergence rate of the PSO algorithm as function of the selected weights as given by Table 1 will also be studied.

\subsection{Proposed localization procedure based on Particle Swarm Optimization algorithm}

PSO algorithm is an adaptive algorithm which is based on cognitive behavior of a given population of individuals [12]. Each individual represents a potential solution. It moves its position in search space and updates its velocity according to its own flying experience and that of its neighbors, aiming for a better position for itself at the next move. This requires the selection of three parameters, two acceleration constants denoted $c_{1}$ and $c_{2}$ and an inertia weight $\mathrm{w}$, which are shown to affect largely convergence of the PSO algorithm. A detailed study on the effects of these parameters and convergence properties of PSO algorithm is given in [13].

The performance of the PSO algorithm in terms of accuracy and computational time has been shown to be better than that of GA based approaches. A multitude of applications have assessed the relevance of the PSO approach. It is able to provide high quality solutions in comparison with experiment or with other known methods $[10,14,15,16]$. Even if there is no clear link to physics or mathematics that could justify rationally this performance, the merit of the PSO algorithm is associated to the fact that it works satisfactorily in practice. 
The direct formulation of beam impact problem enables evaluating the transfer matrices intervening in the nonlinear constrained mathematical program defined by equations (8) and (9). The PSO algorithm implemented under Matlab software package can then be used to find the minimum of the localization problem, equations (8) and (9), which determines the impact zone characteristics $\left(\mathrm{s}_{0}, \mathrm{u}_{0}\right)$. In all the subsequent applications the parameters of the PSO algorithm are fixed at the following values $\mathrm{c}_{1}=0.5, \mathrm{c}_{2}=1.25$ and $\mathrm{w}=0.4$. The random parts are simulated by using the random number generator as provided by the Matlab built-in function rand. Iterations of the PSO algorithm are continued until the maximum number of 100 iterations is achieved.

To study the effect of the number of implemented sensors $\mathrm{N}_{\mathrm{s}}$ and their positions on the ability of the PSO algorithm to converge to the desired solution with enough accuracy, three configurations of sensors implementation are examined. They are chosen among all the possible configurations that can be obtained from the set of sensors shown in figure 1 . Table 2 gives the definition of the configurations that will be tested in the following.

\begin{tabular}{|c|c|c|}
\hline Configuration & Number of sensors & List of sensors \\
\hline 1 & 2 & $\# 2$ and $\# 4$ \\
\hline 2 & 3 & $\# 1, \# 2$ and $\# 3$ \\
\hline 3 & 3 & $\# 1, \# 2$ and $\# 4$ \\
\hline
\end{tabular}

Table 2. Considered configurations for strain sensors implementation

Each configuration of sensors is to be tested according to the selected case of weights coefficients $\alpha_{\mathrm{ij}}$ which are given in Table 1. The objective is to assess the following issues:

- which weighing coefficients $\alpha_{\mathrm{ij}}$ are the best for providing better pressure location solution in terms of accuracy?

- which $\alpha_{i j}$ achieve the best cost effective solution in terms of the number of iterations required to get convergence of the PSO algorithm?

- How the results can change for a given selection of the $\alpha_{\mathrm{ij}}$ with respect to a considered configuration of sensors: number of sensors used and their positions?

- How does behave the PSO algorithm in comparison with the GA?

\section{Results and discussion}


The direct problem of beam impact is considered with the following material and geometric data: $\mathrm{E}=7.06 \times 10^{10} \mathrm{~Pa} ; \mathrm{L}=0.5 \mathrm{~m} ; \mathrm{b}=5 \times 10^{-3} \mathrm{~m} ; \mathrm{e}=5 \times 10^{-3} \mathrm{~m} ; \rho=2660 \mathrm{~kg} \cdot \mathrm{m}^{-3}$; $\xi_{\mathrm{m}}=2 \% ; \mathrm{s}_{0}=0.417 \mathrm{~m} ; \mathrm{u}_{0}=0.0417 \mathrm{~m}$ and $\mathrm{a}=0.25 \mathrm{~m}$. The interval time duration is fixed at $\mathrm{T}_{\mathrm{c}}=1 \mathrm{~s}$. Figure 3 shows the half sine impulse pressure applied to the beam. The maximum pressure is taken to be $10^{5} \mathrm{~Pa}$ and the pulse duration is $\mathrm{T}=0.2 \mathrm{~s}$. This impulse was used to calculate by using the direct problem the normal longitudinal strains at the upper fiber of the beam where the sensors were located.

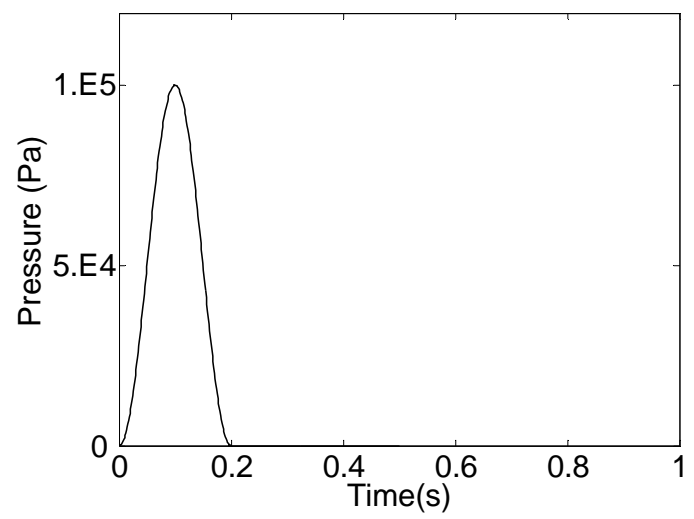

Fig. 3. Time history of the impulse impact pressure

Figure 4 shows convergence of the PSO algorithm iterations for the different values of coefficients $\alpha_{i j}$ as given in Table 1. The fineness function used is that associated to the configuration number 1 of sensors which corresponds to line 2 of Table 2. Figure 5 shows convergence of the PSO iterations for the configuration number 2 of sensors. Figure 6 shows convergence behavior of the PSO algorithm for configuration number 3 which uses the sensors $\# 1$, \#2 and \#4. For comparison with the GA results, figure 7 gives convergence of the solution as function of the iteration number when the fitness function is that associated to combination 3 of Table 1, that is to say the same conditions than those of figure 6.

Figure 4 shows that convergence fails to be reached for all cases when using the two sensors \#2 and \#3. This is true for all combinations of coefficients $\alpha_{i j}$, notice that for the case 2 of these coefficients the converged solution is not very far from the exact solution. But, the need that the localization problem should be solved with high accuracy in order to ease force signal reconstruction impedes to retain this combination of two sensors.

In case of configuration number 2 of sensors where the three sensors $\# 1, \# 2$ and $\# 3$ are used, figure 5 , the two cases 2 and 4 of coefficients $\alpha_{i j}$ succeeded in converging towards the 
exact solution, while cases 1 and 3 fail. Both cases 2 and 4 need about 75 iterations to achieve convergence.

For the last configuration of sensors, combination 3 for which the sensors \#1,\#2 and \#4 are used, figure 6, convergence is reached by the three cases 1,2 and 4 and fails for the case 3 of coefficients $\alpha_{\mathrm{ij}}$. One can notice that the case 2 is the fastest one, since about 38 iterations are sufficient to obtain the exact solution.

Using the GA confirmed that the case 2 of coefficients $\alpha_{\mathrm{ij}}$ is the most favorable one as figure 7 shows that only this case and case 4 can provide the exact solution. However, the case 2 is the best one since the total number of required GA iterations is about 43 against almost the double for the case 4 .

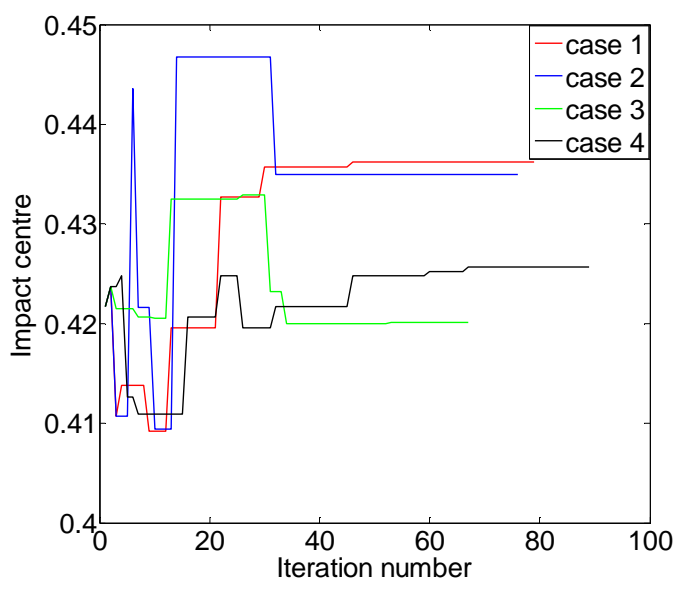

(a)

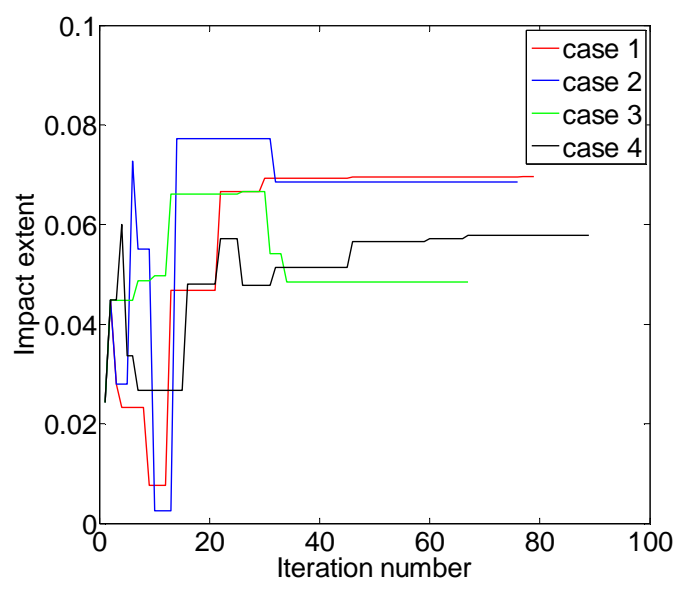

(b)

Fig.4. Configuration 1 of sensors (\#2 and \#3); evolution of impact centre (a) and extent (b) as function of PSO iterations for a given case of coefficients $\alpha_{i j}$

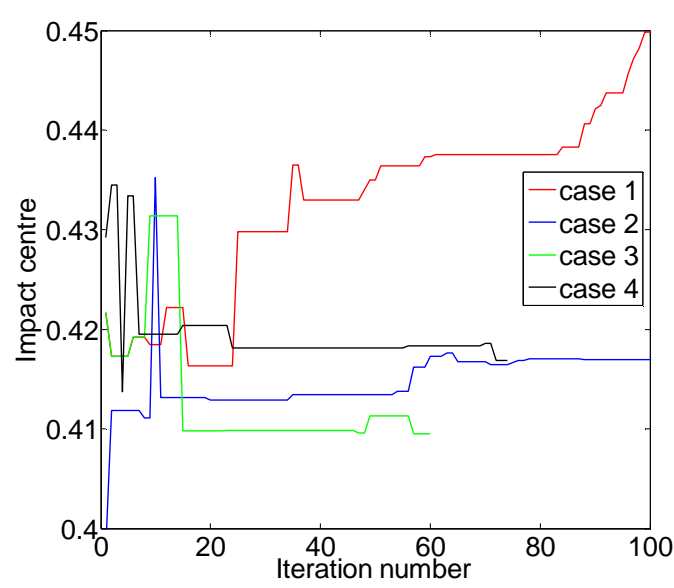

(a)

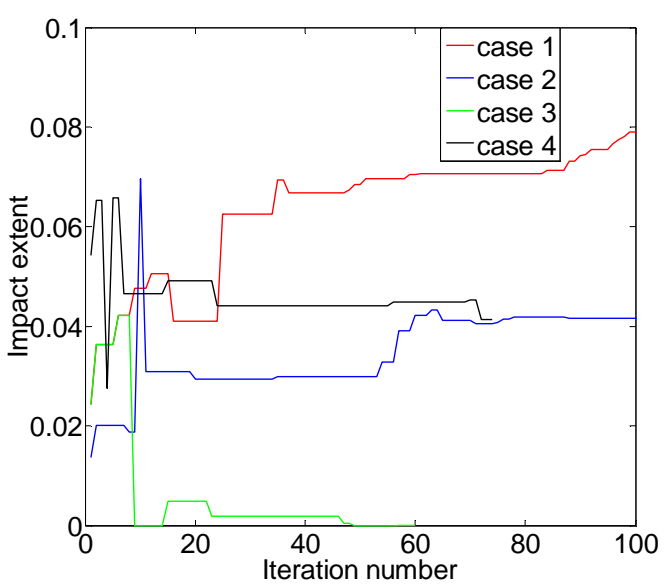

(b)

Fig.5. Configuration 2 of sensors (\#1, \#2 and \#3); evolution of impact centre (a) and extent (b) as function of PSO iterations for a given case of coefficients $\alpha_{\mathrm{ij}}$ 


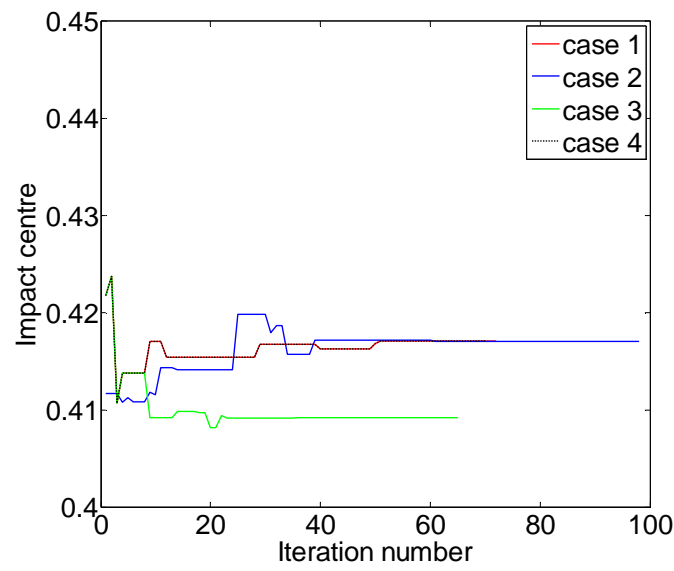

(a)

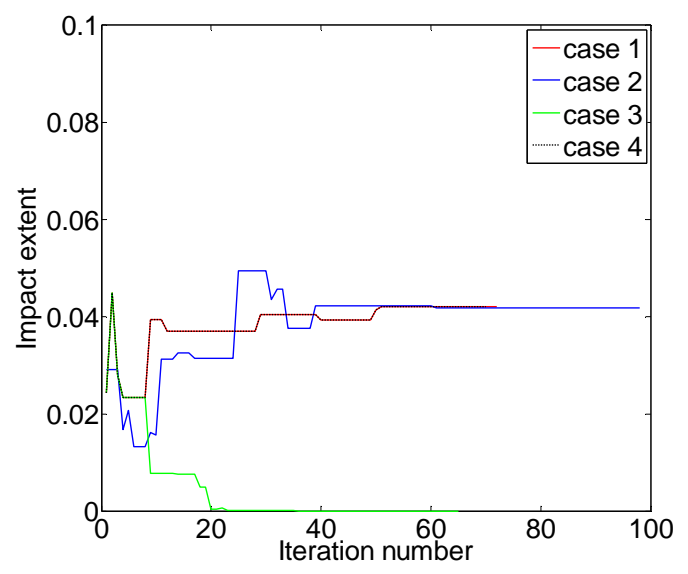

(b)

Fig.6. Configuration 3 of sensors (\#1, \#2 and \#4); evolution of impact centre (a) and extent (b) as function of PSO iterations for a given case of coefficients $\alpha_{i j}$

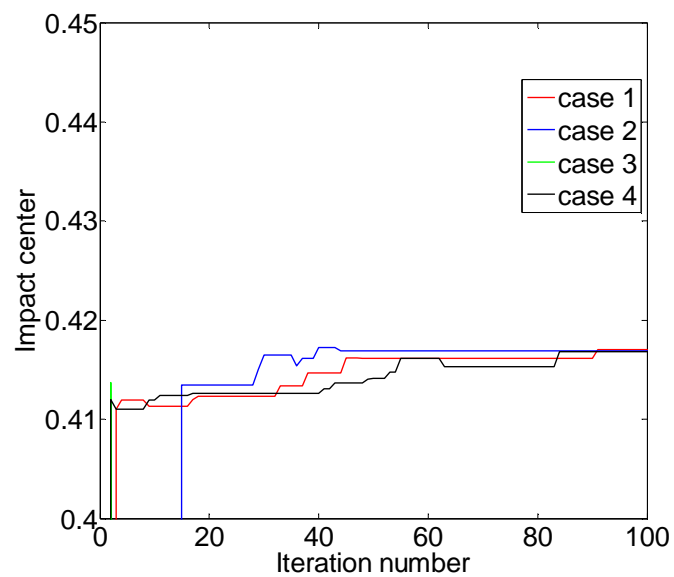

(a)

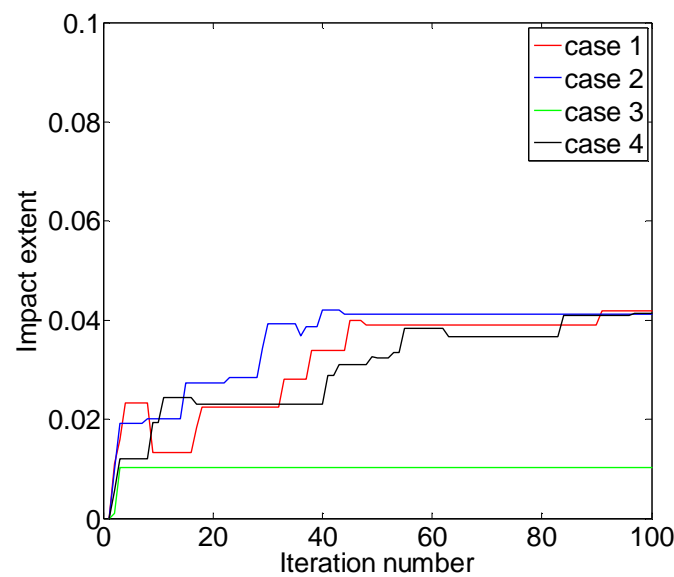

(b)

Fig.7. Configuration 3 of sensors (\#1, \#2 and \#4); evolution of impact centre (a) and extent (b) as function of GA iterations for a given case of coefficients $\alpha_{\mathrm{ij}}$

One can notice then that the coefficients $\alpha_{i j}=\left\|G_{i}(s, u)\right\|^{2}+\left\|G_{j}(s, u)\right\|^{2}$ which correspond to the case 2 constitute the best choice as convergence is always reached when using three sensors and more quickly than for the case 4 of these coefficients. Both the PSO based and the GA algorithms agree on this result.

It should be mentioned that the PSO algorithm admits a net advantage over the GA since this last fails to start iterations normally and early divergence was observed in many occasions. So a lot number of trials were needed in order that iterations reproduce correctly in the process. This is due to the fact that this algorithm handles less effectively constrained optimization problems than does the PSO algorithm and a bad chosen initial population can impede convergence to occur. The main merit of the PSO is that it is more accurate and more 
robust than the GA. As to computational cost, the PSO and the GA were found to require the same order of CPU-time without counting the number of trials needed where this last diverges.

From the above discussion one can make the following recommendations.

- Two sensors are not sufficient to perform accurately solution of the localization problem. Weight coefficients defined by the case 2 of Table 1 provide a result which is not very far from the exact solution, while all the others fail by a large amount.

- When using three sensors the coefficients $\alpha_{i j}=\left\|G_{i}(s, u)\right\|^{2}+\left\|G_{j}(s, u)\right\|^{2}$ corresponding to the case 2 of Table 2 constitute the best weighting combination. They provide the exact solution with the fewest number of iterations.

- The PSO algorithm was found to be more robust providing repeatable results unlike the GA which fails to converge sometimes, depending on the initial population used.

\section{Conclusions}

An optimization technique based on PSO algorithm was used to achieve force location in case of an elastic structure having the form of a beam when subjected to a sudden impact. The impact was assumed to be non punctual and resulting in a distributed uniform pressure over the impacted zone. The proposed methodology took into account both the effect of the number of sensors used and their positions. It has enabled to determine the most favorable combination of weighting coefficients that are introduced to remove the parasitic solutions appearing when formulating the inverse localization problem by means of the Maxwell-Betti theorem. This combination proved to be effective in performing regularization of the force location problem when using a combination of three sensors. It enabled to achieve good accuracy and minimum computational time. Besides, the PSO algorithm revealed to be more effective in comparison with the GA, since it was found to be less sensitive to initialization and requiring almost the same CPU-time than this last.

It should be noticed that the above remarks hold for the impact problem considered in this case study. Further investigation is required in order to assess that the weighting coefficients found in this work continue to guarantee convergence of the PSO algorithm when applied to the inverse location problem for other kind structures.

\section{References}

[1] R. Aster, B. Borchers, and T. Clifford. Parameter Estimation and Inverse Problems, Second Edition, Elsevier, 2012. 
[2] R. Seydel, F.K. Chang, Impact Identification of Stiffened Composite Panels. 1: System Development. Smart Materials and Structures, 10(2) (2001) 354-369.

[3] R. Seydel, F.K. Chang, Impact Identification of Stiffened Composite Panels. 2: Implementation Studies. Smart Materials and Structures, 10(2) (2001) 370-379.

[4] C.S. Yen, E. Wu, On the Inverse Problem of Rectangular Plates Subjected to Elastic Impact, Part 1: Method Development and Numerical Verification, Journal of Applied Mechanics 62 (1995) 692-698.

[5] J.F. Doyle, Modern Experimental Stress Analysis, completing the solution of partially specified problems, John Wiley \& Sons Ltd, Chichester, West Sussex, England, 2004.

[6] K. Choi, F.K. Chang, Identification of foreign object impact in structures using distributed sensors, Journal of Intelligent Material Systems and Structures 5 (1994) 864-869.

[7] M.T. Martin J.F. Doyle, Impact force location in frame structures, International journal of impact engineering 18 (1996) 79-97.

[8] T. Kundu, S. Das, S.A. Martin and K.V. Jata, Locating point of impact in anisotropic fiber reinforced composite plates. Ultrasonics 48 (3) (2008) 193-201.

[9] D. Liang, S.F. Yuan and M.L. Liu. Distributed coordination algorithm for impact location of preciseness and real-time on composite structures. Measurement 46 (2013) 527-536.

[10] S. Suresh, P.B. Sujit, A.K. Rao, Particle swarm optimization approach for multiobjective composite box-beam design. Composite Structures 81 (2007) 598-605.

[11] L. Meirovitch. Elements of Vibration Analysis. Mc-Graw Hill Inc., Singapore, 1986.

[12] J. Kennedy, R.C. Eberhart, Y. Shi. Swarm Intelligence. Morgan Kaufmann Publishers, 2001.

[13] I.C. Trelea. The particle swarm optimization algorithm: convergence analysis and parameter selection. Inform Process Lett 85 (2003) 317-325.

[14] Y. Dong, J. Tang, B. Xu and D. Wang. An Application of Swarm Optimization to Nonlinear Programming. Computers and Mathematics with Applications 49 (2005) 16551668 .

[15] M. Sadeghierad, A. Darabi, H. Lesani and H. Monsef. Optimal design of the generator of microturbine using genetic algorithm and PSO. Electrical Power and Energy Systems 32 (2010) 804-808.

[16] S.A. Taher, A. Karimian and M. Hasani. A new method for optimal location and sizing of capacitors in distorted distribution networks using PSO algorithm. Simulation Modelling Practice and Theory 19 (2011) 662-672. 